\title{
Utility of serum ADA estimation in the diagnosis of extrapulmonary tuberculosis
}

\section{Authors \\ Prashant Chikkahonnaiah ${ }^{1}$, Surabhi Jaggi ${ }^{2}$, Bela Goyal ${ }^{3}$, Kranti Garg ${ }^{4}$, Seema Gupta ${ }^{5}$, Shivani Jaswal ${ }^{6}$, Kamaljeet Kaur $^{7}$}

${ }^{1}$ Assistant Professor, Dept of Pulmonary Medicine, Mysore Medical College and Research Institute Mysore ${ }^{2}$ PG JR, Department of Pulmonary Medicine, ${ }^{3}$ Assistant Professor, Department of Biochemistry, ${ }^{4}$ Assistant Professor, Department of Pulmonary Medicine, ${ }^{5}$ Assistant Professor, Department of Biochemistry,

${ }^{6}$ Professor, Department of Biochemistry, ${ }^{7}$ Biochemist, Department of Biochemistry 2,3,4,5,6,7 Government Medical College \& Hospital, Chandigarh, India

\section{Corresponding Author}

\section{Dr Surabhi Jaggi}

PG JR, Department of Pulmonary Medicine, Government Medical College \& Hospital, Chandigarh, India Email: surabhijaggi@gmail.com

\begin{abstract}
Background: Adenosine deaminase (ADA) has largely been studied in body fluids for the diagnosis of extra pulmonary tuberculosis (EPTB). Serum ADA has been used as non-invasive assay for Pulmonary TB. The aim of the present study was to test the utility of serum ADA for the diagnosis of EPTB.

Methods: A retrospective analysis of serum ADA values from 122 patients was carried out. The patients were divided into three groups based on their diagnosis i.e. Group A: pulmonary TB patients, group B: Extra pulmonary TB patients and Group C: patients with other respiratory illnesses. Nonparametric tests were performed to compare the groups. Receiver operating curve was plotted to determine the cut-off for knowing the sensitivity and specificity of serum ADA.

Results: The mean serum ADA levels in patients of TB showed significantly higher values than disease controls (other respiratory illnesses). Although, the levels of mean ADA were higher in group B as compared to group $A$, it was not statistically significant. ROC showed sensitivities of $73.7 \%$ and $89.5 \%$ for group $A$ and group $B$ respectively at the cut-off of 17.5 and a high AUC of 0.814 and 0.945 for group A and group B respectively when compared to disease controls (group $C$ ).

Conclusions: Serum ADA has a potential to be used as surrogate marker for the diagnosis of sputum smear negative and extra pulmonary $T B$.

Keywords- Serum Adenosine deaminase, Pulmonary tuberculosis, Extra pulmonary tuberculosis.
\end{abstract}

\section{Introduction}

Tuberculosis (TB) is one of the most common and dreaded disease worldwide. It accounts for around 2 million deaths per year ${ }^{1}$. Tuberculosis can present as either Pulmonary TB (PTB) or extra Pulmonary TB
(EPTB), where TB occurs at sites other than lungs and includes pleural, pericardial, meningeal, gastrointestinal and genitourinary tract TB.

The diagnosis of extra pulmonary $\mathrm{TB}$ is usually made by direct or indirect methods. Direct methods 
involve demonstration of TB bacilli by microscopy, culture or molecular methods. Indirect methods involve detection of humoral or cell mediated immune response of host to mycobacterial antigen or detection of biomarkers like Adenosine deaminase (ADA), Interferon $\gamma$ (IFN $\gamma$ ) etc. ${ }^{2}$

Low sensitivity of microscopy and staining (0$40 \%$ ), delayed diagnostic time (6-8 wks) for gold standard culture method, invasiveness of histological techniques and paucibacillary nature of extra pulmonary TB- all perturb their utility for EPTB diagnosis. ${ }^{3}$ Nucleic acid amplification techniques (NAAT) is expensive and requires sophisticated instrumentation. Antibody based tests and Cell Mediated Immunity based tests only provide supportive evidence.

So, the need of the hour is to develop a test which is rapid, cost effective, easy to perform in a resource poor country like India to reduce morbidity and mortality associated with extra pulmonary TB. Levels of adenosine deaminase (ADA) in body fluids especially pleural fluid has proven to be highly sensitive and specific for diagnosis of extra pulmonary TB. ${ }^{4-7}$ However, in some cases diagnosis of EPTB may require invasive procedures like FNAC or biopsy. So, serum ADA seems to be a better alternative to fluid ADA for the diagnosis of EPTB.

ADA being an enzyme of purine nucleotide metabolism deaminates adenine to inosine. It has been shown that mycobacterial antigen stimulates production of ADA in lymphocytes and monocytes. ADA is required for differentiation as well as proliferation of these cells. ${ }^{8,9}$ Limited studies are available for the utility of serum ADA estimation for the diagnosis of EPTB .

The aim of the present study was to find the utility of using serum ADA for the diagnosis of EPTB.

\section{Methods}

The present study was a retrospective observational pilot study conducted at a Tertiary Care Hospital of North India. The result of 122 patients admitted or attending outpatient department of the department of pulmonary medicine who were investigated for different respiratory disorders were analysed for serum ADA levels. Based on the clinical history, the patients were grouped as follows:

Group A (N=19): Newly diagnosed pulmonary TB patients, both sputum smear positive and negative.

Group B ( $\mathbf{N = 2 2 ) : ~ N e w l y ~ d i a g n o s e d ~ E P T B ~ p a t i e n t s . ~}$

Group C (N=82): Respiratory illnesses other than TB.

Patients with diabetes mellitus, those on corticesteroids or anti-tubercular treatment or with evidence of hepatic or renal impairment were excluded.

The serum ADA levels were determined using Diazyme Adenosine Deaminase assay kit (Diazyme laboratories, USA) that determines ADA level by kinetic enzymatic method on Random access Chemistry Autoanalyzer Modular P-800.

\section{Statistical Analysis}

Statistical analysis was performed using SPSS v.16.0 (SPSS Inc, Chicago, IL, USA) for MSWindows. Levey's test was performed to test for the homogeneity of variance. Non parametric KruskelWallis test was performed to determine any statistically significant difference between all the groups. A two sample Kolmogorov Smirnov test was performed to compare two independent groups with each other. ROC curve was plotted to determine the cut-off value for ADA test and Area under curve (AUC) was used to determine the discriminatory ability of the assay to distinguish between TB (pulmonary or extra pulmonary) from non TB respiratory illnesses. Statistically significant different was calculated at $\mathrm{p}<0.05$.

\section{Results}

The mean levels of serum ADA were found to be significantly higher in the patients diagnosed with TB in comparison to the ones who were suffering from any other respiratory illnesses $(\mathrm{p}<0.05)$. The test results showed that mean value of Serum ADA in patients of Group B (EPTB) was higher than in patients of Group A (PTB). (Table 1) However, no significant difference was observed between these two groups. However, patients of Group C (Respiratory illnesses other than TB) had significant 
lower value of serum ADA than both the TB groups (Group A \& B).

Table 1: Levels of ADA activity in sputum smear positive (Group A), sputum smear negative with extrapulmonary TB (Group B) and other respiratory illnesses (Group C)

\begin{tabular}{|l|c|c|c|c|}
\hline Groups & Mean \pm SD & SEM & \multicolumn{2}{|c|}{$\begin{array}{r}\text { 95\% confidence } \\
\text { interval }\end{array}$} \\
\hline & & & $\begin{array}{c}\text { Lower } \\
\text { bound }\end{array}$ & $\begin{array}{c}\text { Upper } \\
\text { bound }\end{array}$ \\
\hline Group A (N=19) & $26.89 \pm 10.4^{*}$ & 2.39 & 21.87 & 31.91 \\
\hline Group B (N=24) & $37.12 \pm 13.6^{*}$ & 2.78 & 31.36 & 42.88 \\
\hline Group C (N=79) & $15.7 \pm 4.8$ & 0.54 & 14.65 & 16.83 \\
\hline
\end{tabular}

*Indicates statistically significant difference at $\mathrm{P}<0.05$ by non- parametric two tailed Kolmogorov-Smirnov test.

ROC plots showed a high discriminatory power of ADA assay for diagnosis of TB over non TB respiratory illnesses with AUC of 0.945 and 0.814 for PTB and EPTB respectively. (Figure-1) According to the coordinates of ROC curve, at the cut-off value of 17.5 , assay showed sensitivity of $73.7 \%$ and specificity of $64.6 \%$ for PTB. For EPTB group, at the cut-off value of 17.5 , sensitivity of $89.5 \%$ and specificity of $64.6 \%$ was observed.

Figure 1: ROC plot showing Area under curve (AUC) of groups A, B

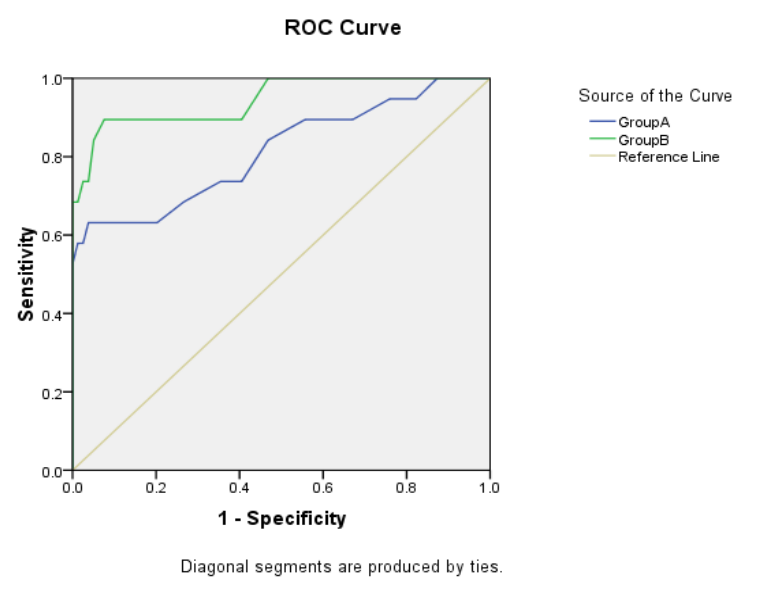

\section{Discussion}

Despite the huge advances in the diagnostic techniques for the diagnosis of TB, the challenge of developing a novel, cost effective, accurate bio marker based point of care test still prevails. Traditional assays lack sensitivity/specificity or are time consuming or are invasive precluding their utility in resource poor set up. ADA is an enzyme whose activity increases when $\mathrm{T}$ lymphocytes are stimulated by mycobacterium. The ADA then aids in the differentiation and proliferation of these $\mathrm{T}$ lymphocytes. The importance of ADA in body fluids has already been established for the diagnosis of pleural and other extra pulmonary TB, where sputum often leads to inconclusive results. ${ }^{4-7}$ This observation of increased ADA activity in body fluids is believed to be due to migration of $T$ lymphocytes to the effusion sites. ${ }^{9}$ However, very limited studies are available for ADA levels in serum. The ease of venipuncture over access to other body fluids certainly appears to be more advantageous

In the present study, it was observed that $\mathrm{TB}$ patients showed higher serum ADA levels as compared to non TB respiratory illnesses group indicating increased activity of ADA in TB. This observation validates the observation by Salmanzadeh $\mathrm{S}$ et al, where they found higher serum ADA levels in pulmonary TB than non TB respiratory illnesses.10 Studies by Afrasibran et al and Samy Atta also showed similar results, where they compared serum ADA levels of pulmonary TB patients with other respiratory illnesses. ${ }^{11,12}$ However, this observation is in contrast with the study by Aliasgarh Farazi that showed low sensitivity of serum ADA assay for differentiating TB from other respiratory illnesses and thus disapproved its utility for TB diagnosis. ${ }^{13}$

The finding of higher activity in EPTB than PTB patients in the present study could be due to the higher $\mathrm{T}$ lymphocytes activity in that group. However, since in EPTB group, tubercular foci may not be proximal to the bronchial tract, sputum smear examination is negative. This appears to be quite promising as this could obviate the need of invasive tests or expensive tests for the diagnosis of this group. This corroborates with the studies by Agarwal et al and Anil Chander where they showed higher serum ADA levels in sputum smear negative pulmonary TB patients than other lung diseases like pneumonia, pulmonary abscess, lung cancer etc. ${ }^{14,15}$ 
Moreover ROC curve clearly shows high discriminatory power of the assay for both smears positive or negative TB over other respiratory illness and reasonably high sensitivity at the cut-off of 17.5. However, lesser number of subjects might have led to a higher standard deviation and thus a low specificity.

This study was one of the first of its kind that compared serum ADA levels in EPTB over other respiratory illnesses. However, there were few limitations of the study. This study was the pilot study with small number of subjects and involved a single geographical area. Further studies with larger group and that can categorize the patient based on their bacillary load should be planned and executed for validation of the result.

\section{Conclusion}

To conclude our study indicates that serum ADA can be used as a supportive surrogate marker for difficult to diagnose smear negative and extra pulmonary $\mathrm{TB}$ and may also be used to differentiate TB from other respiratory illnesses. However, further validation with larger studies must be done.

\section{References}

1. WHO. Global tuberculosis report 2013. Tuberculosis -epidemiology. WHO Press, World Health Organization, 20 Avenue Appia, 1211 Geneva 27, Switzerland. 2013: 1-11.

2. Purohit M, Mustafa T, Laboratory Diagnosis of Extra-Pulmonary Tuberculosis (EPTB) In Resource - Constrained Setting: State of The Art, Challenges And The Need, Journal of Clinical And Diagnostic Research, Apr 2015; 9(4):EE01-EE06.

3. Harries AD, Gausi F, Chimzizi R, Salaniponi FM. Characteristics and outcome of tuberculosis patients whose sputum smears are positive at or after 5 months of treatment. Int J Tuberc Lung Dis. 2004 Mar;8(3):384-7.

4. Greco S, Girardi E, Masciangelo $\mathrm{R}$ et al Adenosine Deaminase and interferon gamma measurements for the diagnosis of tuberculous pleurisy: a metaanalysis. Int $\mathrm{J}$ Tuberc Lung Dis 2003; 7 (8): 777-86.

5. Piras MA, Gakis C, Budroni M, Andreoni G. Adenosine deaminase activity in pleural effusions: An aid to differential diagnosis. Br Med J. 1978; 2:1751-2.

6. Tuon FF, Silva VI, Almeida GM, Antonangelo L, Ho YL. The usefulness of adenosine deaminase in the diagnosis of tuberculous pericarditis. Rev Inst Med Trop Sao Paulo. 2007;49:165-70.

7. Saleh MA, Hammad E, Ramadan MM, Abd El-Rahman A, Enein AF. Use of adenosine deaminase measurements and QuantiFERON in the rapid diagnosis of tuberculous peritonitis. J Med Microbiol. 2012;61:514-9.

8. Kuyucu N, Karakurt C, Bilaloğlu E, Karacan $\mathrm{C}$, Teziç T. Adenosine deaminase in childhood pulmonary tuberculosis: Diagnostic value in serum. J Trop Pediatr. 1999; 45:245-7.

9. Barua R, Hossain M. Adenosine deaminase in diagnosis of tuberculosis: a review. Anwer Khan Mod Med Coll J. 2014; 5:43-8. 10. Salmanzadeh S, Tavakkol H, Bavieh K, Alavi SM. Diagnostic Value of Serum Adenosine Deaminase (ADA) Level for Pulmonary Tuberculosis. Jundishapur J Microbiol. 2015;8(3):e21760

11. Afrasiabian S,Mohsenpour B, Bagheri KH, Sigari N, Aftabi K. Diagnostic value of serum adenosine deaminase level in pulmonary tuberculosis, J. Res. Med. Sci. 18 (3 (March)) (2013) 252-254.

12. Atta S, Kassem A, Elhadidi A, El Esawy H. The diagnostic value of adenosine deaminase activity in pulmonary tuberculosis: Comparison between sputum and serum. Egypt J Chest Dis Tuberc.2015;64:103-107.

13. Farazi A, Moharamkhani A, Sofian M. Validity of serum adenosine deaminase in diagnosis of tuberculosis. Pan Afr Med J. 2013; 15:133. 
14. Agarwal MKN, Mukerji PK, Srivastava VML. A study of serum adenosine deaminase activity in sputum negative patients of pulmonary tuberculosis. Ind $\mathrm{L}$ Tub. 1991; 38:139.

15. Chander A, Shrestha CD. Diagnostic Value of Serum Adenosine Deaminase Levels in Sputum Smear Negative Pulmonary Tuberculosis Patients In Nepalese Population, Asian Pacific Journal of Tropical Biomedicine, 2012: S1896 - S1899. 